\title{
OPINION
}

\section{Adding a Medicine to the WHO Model List of Essential Medicines}

\author{
SP Kishore ${ }^{1}$ and BJ Herbstman ${ }^{1}$
}

\begin{abstract}
Although the concept of essential medicines is not new, the processes that lead to placement of these medicines on the World Health Organization Model List of Essential Medicines are not well understood. Here, we describe the process by which we added a class of drugs, statins, to the Model List to help combat the growing global epidemic of cardiovascular disease.
\end{abstract}

In 2005, there were no lipid-lowering drugs on the World Health Organization (WHO) Model List of Essential Medicines. That same year, cardiovascular disease (CVD) was responsible for nearly $30 \%$ of deaths worldwide, with nearly $80 \%$ of these deaths occurring in developing countries. ${ }^{1}$ The unprecedented burden of CVD in poor countries-partially attributable to higher rates of urbanization, smoking, and sedentary lifestyles-is only expected to worsen. It is projected that by 2010 CVD will be the leading cause of death in developing countries. ${ }^{2}$ With the current and predicted state of CVD in these regions, we, two medical students, thought it was especially important for the major class of lipid-lowering drugs-statins-to become accessible as soon as possible.

We chose to improve accessibility to statins by qualifying this class of drugs on the WHO Model List of Essential Medicines, which includes drugs that, according to the WHO, "satisfy the priority health care needs of the population." Critically, anyone can submit an application for a medicine to be added to or deleted from the list. First published in
1977 and revised every two years by an Expert Committee, the list catalogs critical medicines and guides purchasing decisions made by governments and many organizations. For example, major nongovernmental organizations and United Nations agencies limit the drugs that they purchase for donation to those on the list. The list also functions as an advocacy tool. Once medicines have been added in accordance with a population's health priorities, international pressure can drive down prices and increase access to these medicines, even if they are on patent. The addition of antiretrovirals in 2002, for instance, was seen as a major move to encourage governments in countries hard hit by HIV/AIDS to expand distribution of these vital drugs. ${ }^{3}$ The list is meant not to replace individual, national essential medicines lists, but rather, to be used as a guide to inform purchasing decisions in the context of national health agendas.

Although the concept of essential medicines is not new, the processes that lead to placement of these medicines on the Model List are not well understood. The general process of adding statins to the list is summarized in Figure 1. Below is a brief roadmap of how we (i) assessed efficacy and safety of the drugs in a global population, (ii) quantified and modeled cost and cost-effectiveness in low- and middle-income countries, and (iii) identified a reliable, multisource generic supply of statins. The recent patent expiration of simvastatin in the United States led us to choose this statin as an example of the class.

\section{Adding simvastatin to the WHO Model List}

Assess efficacy and safety of simvastatin in a global population. Many of the statin trials-e.g., the Heart Protection Study, the Scandinavian Simvastatin Survival Study, AFCAPS/TexCAPS (Air Force/ Texas Coronary Atherosclerosis Prevention Study) - have taken place in Europe or the United States, with predominantly Caucasian, elderly male populations in developed countries. Although the current data on the effect of a statin on various races and ethnicities are not conclusive, there is fairly robust evidence that the correlation between elevated cholesterol levels and coronary disease holds true for all ethnic groups. ${ }^{4-6}$ In particular, the greater the reduction in low-density lipoprotein cholesterol, the greater the reduction in vascular risk-a decrease of $1 \mathrm{mmol} / \mathrm{l}$ over 5 years reduces major vascular events by $23 \%$ (ref. 7). Other reports, however, indicate that response to statins differs widely across certain populations. Asians, particularly Chinese, require half the dose that Caucasians do, owing to differences in drug metabolism. ${ }^{8,9}$ Japanese demonstrate a sustained $20 \%$ reduction in low-density lipoprotein cholesterol on one-fourth the recommended dose of simvastatin. ${ }^{10}$ The differences in drug efficacy for subgroups with varying CVD risk factors (men, women, the elderly, and those with diabetes) have been

${ }^{1}$ Weill Cornell Medical College, New York, New York, USA. Correspondence: SP Kishore (sunny.kishore@gmail.com) 


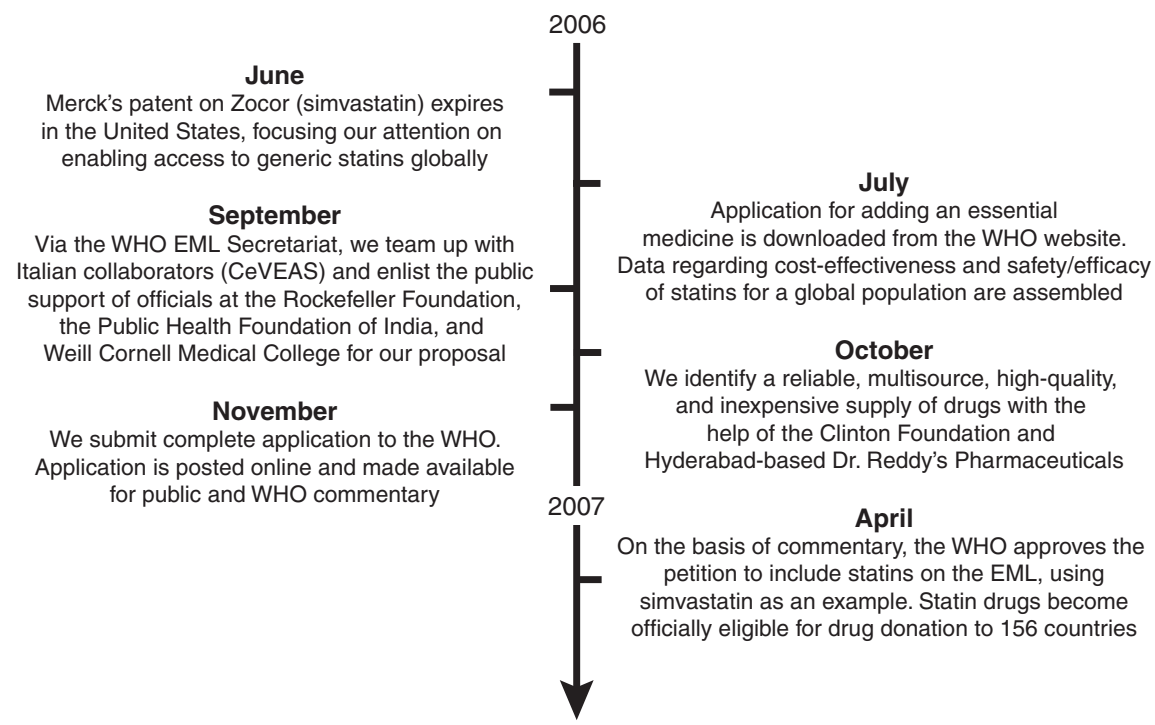

Figure 1 Adding statins to the 15th WHO Model List of Essential Medicines. The key events in our bid to have statins listed are highlighted. The formal application form to add or delete an essential medicine is available at http://www.who.int/medicines/publications/essentialmedicines. Our complete submission can be viewed at http://archives.who.int/eml/expcom/expcom15/applications/newmed/statins/statins. pdf. CeVEAS, Centre for the Evaluation of the Effectiveness of Health Care (Modena, Italy); EML, Essential Medicines List; WHO, World Health Organization.

highlighted. Moreover, whereas various statins are metabolized differently and feature different drug interactions, there are few discernible differences in the efficacy of different statins. ${ }^{11}$ Therefore, the choice of statin should ultimately be left to individual nations. To ensure safety, we recommended that patients seek medical care in the first year of treatment to be monitored for liver-function impairment and evaluated for rhabdomyolysis-adverse effects that are consistent throughout populations. Current information on ethnic and geographic cardiovascular risk profiles is being compiled by Inter-Heart (the WHO and the World Heart Federation). ${ }^{12}$

Determine cost and cost-effectiveness of simvastatin globally. After simvastatin's US patent expired on 23 June 2006 (ref. 13), a global decrease in the drug's price was expected, driven by price reductions in the United States. In the United States, several generic manufacturers would be permitted to compete for market share beginning in January 2007. The projected price for statins was expected to be $\$ 40$ per year of treatment. Our data corresponded with the International Drug Price Indicator Guide published by the Management Sciences for Health (MSH) group. This guide catalogs all tender agreements between pharmaceutical firms and national governments concerning bulk purchase of medicines. We understood from our discussions with firms and managers of the MSH guide that some countries have in-house production facilities that may make production of one generic statin more feasible and cost-effective than another. For example, although generic lovastatin is considered a lower-potency statin (30 mg lovastatin $=20 \mathrm{mg}$ simvastatin), it has the advantage of lower price in some regions, as low as $\$ 7$ per year of treatment. ${ }^{14}$

Many of the available data-particularly those published by the Disease Control Priorities Project-support the notion that a statin is cost-effective for secondary prevention according to $\mathrm{WHO}$ standards. ${ }^{15}$ These standards define cost-effectiveness mathematically as a cost per disability-adjusted life year that is less than three times the gross national income per capita. Recent data indicate that by this definition, a multidrug regimen-a statin, an aspirin, a $\beta$-blocker, and an angiotensin-converting enzyme inhibitor or calcium channel blockeris cost-effective for primary prevention in high-risk individuals in the developing world. ${ }^{16}$
Determine whether a reliable, multisource supply of simvastatin exists. Surveillance of high-quality generic forms of simvastatin was done by a colleague of ours at the Clinton Foundation. Global manufacturers were selected from the Directory of World Chemical Producers, a publicly accessible sourcing database, using "simvastatin" as the query term. ${ }^{17}$

The data indicated that several countries had reliable, multisource supplies of registered generic medicines. A full catalog of the manufacturers and contact information was submitted to the $\mathrm{WHO}$ as an appendix to our proposal. As a test case, one of the simvastatin manufacturers (Dr. Reddy's Pharmaceuticals, Hyderabad, India) was asked to provide detailed information on manufacture protocol, quality assurance, and pricing. All general information on the suppliers and specific information on manufacture, quality assurance, and product registration was sent to the WHO.

\section{Discussion and summary}

The proposal was submitted to the WHO in the fall of 2006 and was approved by April 2007. Our work is a small example of the work that health-care professionals and students can perform to enhance access to medicines. We believe that health-care professionals and students should monitor the WHO Model List on a continual basis in order to prepare thoughtful applications to add or delete medicines. Our experience illustrates that the Expert Committee values scholarship and evidence-based applications over the prestige of the authors. Several important drugs still missing from the current list (the 15th version)-e.g., clarithromycin, which is especially relevant in the context of Helicobacter pylori eradication-require applications for inclusion.

\section{ACKNOWLEDGMENTS}

We thank Patricia Mongelia, Helen-Ann Brown, and Paula Trushin for expert assistance, and Marcus M. Reidenberg and Derek Yach for critical comments on the manuscript. This work was supported by National Institutes of Health Medical Scientist Training Program grant GM07739 and the Paul and Daisy Soros Fellowship to S.P.K. The funders were not involved in the decision to submit the article or in its preparation. 


\section{CONFLICT OF INTEREST}

The authors declared no conflict of interest.

() 2009 ASCPT

1. Gaziano, T.A. Cardiovascular disease in the developing world and its cost-effective management. Circulation 112, 3547-3553 (2005).

2. World Health Organization. Shaping the Future (World Health Organization, Geneva, 2003).

3. Schwartlander, B., Grubb, I. \& Perriens, J. The 10-year struggle to provide antiretroviral treatment to people with HIV in the developing world. Lancet 368, 541-546 (2006).

4. Zhang, X., Patel, A., Horibe, H., Wu, Z., Barzi, F., Rodgers, A. et al. Cholesterol, coronary heart disease, and stroke in the Asia Pacific region. Int. J. Epidemiol. 32, 563-572 (2003).

5. Verschuren, W.M., Jacobs, D.R., Bloemberg, B.P., Kromhout, D., Menotti, A., Aravanis, C. et al. Serum total cholesterol and long-term coronary heart disease mortality in different cultures. Twentyfive-year follow-up of the seven countries study. JAMA 274, 131-136 (1995).

6. Cai, J., Pajak, A., Li, Y., Shestov, D., Davis, C.E., Rywik, S.et al. Total cholesterol and mortality in China, Poland, Russia, and the US. Ann. Epidemiol. 14, 399-408 (2004).

7. Baigent, C., Keech, A., Kearney, P.M., Blackwell, L., Buck, G., Pollicino, C. et al. Efficacy and safety of cholesterol-lowering treatment: prospective meta-analysis of data from 90,056 participants in 14 randomised trials of statins. Lancet 366, 1267-1278 (2005).

8. Tan, C.E., Loh, L.M. \& Tai, E.S. Do Singapore patients require lower doses of statins? The SGH Lipid Clinic experience. Singapore Med.J. 44, 635-638 (2003).

9. Muck, W., Unger, S., Kawano, K. \& Ahr, G. Interethnic comparisons of the pharmacokinetics of the HMG-CoA reductase inhibitor cerivastatin. $\mathrm{Br}$. J. Clin. Pharmacol. 45, 583-590 (1998).

10. Matsuzawa, Y., Kita, T., Mabuchi, H., Matsuzaki, M., Nakaya, N., Oikawa, S. et al. Sustained reduction of serum cholesterol in low-dose 6-year simvastatin treatment with minimum side effects in 51,321 Japanese hypercholesterolemic patients. Circ. J. 67, 287-294 (2003).

11. Law, M.R., Wald, N.J. \& Rudnicka, A.R. Quantifying effect of statins on low density lipoprotein cholesterol, ischaemic heart disease, and stroke: systematic review and meta-analysis. BMJ 326, 1423 (2003).

12. Ounpuu, S., Negassa, A.\& Yusuf, S. INTER-HEART: a global study of risk factors for acute myocardial infarction. Am. Heart J. 141, 711-721 (2001).

13. US Food and Drug Administration. FDA approves generic simvastatin. FDA News <http://www.fda. gov/bbs/topics/NEWS/2006/NEW01394.html> (23 June 2006).

14. Management Sciences for Health (MSH). International Drug Price Indicator Guide < $\mathrm{http}$ :// erc.msh.org $>$ (2007).

15. Gaziano, T.A., Reddy, K.S., Paccaud, F., Horton, S. \& Chaturvedi, V. Cardiovascular Disease (World Bank Group, Washington, DC, 2006).

16. Gaziano, T.A., Opie, L.H. \&Weinstein, M.C. Cardiovascular disease prevention with a multidrug regimen in the developing world: a cost-effectiveness analysis. Lancet 368, 679-686 (2006).

17. Chemical Information Services. Directory of World Chemical Producers <http://www. chemicalinfo.com/products/dwcp/dwcp overview.htm>.
Model-Based

Benefit-Risk Assessment: Can Archimedes Help?

\author{
R Krishna ${ }^{1}$
}

In December 2008, the US Food and Drug Administration issued a
new draft Guidance for Industry on Diabetes Mellitus-evaluating cardiovascular risk in new antidiabetic therapies to treat Type 2 diabetes. This guidance comes at a time when recent discussions have focused on delineation of cardiovascular risk reduction for new antidiabetic drugs. Computational tools that can enable early prediction of cardiovascular risk are reviewed with specific reference to Archimedes (Kaiser Permanente), with an aim of proposing a model-based solution and enabling decisions to be made as early as possible in the drug development value chain.
In a meeting of the US Food and Drug mittee for Endocrinologic and Metabolic Drugs held 1-2 July 2008, an expert panel recommended that developers of antidiabetic drugs perform larger outcome studies to fully delineate reduction of cardiovascular risk as part of the drug development program. If the recommendation is accepted by the FDA, this would be a landmark shift in the regulatory use of surrogate end points, in this case, of glycosylated hemoglobin (HbA1c). It is worth exploring whether the FDA's own Critical Path Initiative offers a model-based solution to the larger question at hand, namely, whether we can meaningfully predict cardiovascular risk on the basis of the clinical studies that are typically done to support regulatory submissions of new drug candidates. Importantly, the question remains whether we can bring critical decisions earlier in the new drug development process, perhaps in phases I-II, to preserve the economic and scientific impact by ensuring that compounds with optimal balance of risk and benefit are developed. This would mean that the Administration (FDA) advisory com- probability that a certain drug development candidate can succeed on the target product profile is predicted according to decision-analytical paradigms.

Several discrete-event simulation tools are available for predicting cardiovascular risk early in drug development. One is the Archimedes model, a mechanistic modeling platform that incorporates pathways related to diabetes, hypertension, obesity, coronary artery disease, congestive heart failure, metabolic syndrome, and other disorders. The physiology-based platform is an excellent avenue to predicting cardiometabolic risk in general. The model incorporates data from many sources, primarily clinical trials and the vast amount of epidemiological data. ${ }^{1-3}$

Although the model is geared primarily toward assessment of the cost-effectiveness of a lifestyle-modification program used in the Diabetes Prevention Program, the model is a useful tool with which to understand risk assessment in the diabetic patient population. In particular, it is an object-by-object and person-by-person simulation that is based on physiology and pathophysiology.
${ }^{1}$ Merck Research Laboratories, Merck \& Co., Inc., Whitehouse Station, New Jersey, USA. Correspondence: R Krishna (rajesh_krishna@merck.com)

doi:10.1038/clpt.2008.240 